\title{
DESESTABILIZAÇÃO DE UMA SUSPENSÃO COLOIDAL DE DIÓXIDO DE TITÂNIO
}

\author{
L. D.C. FREITAS ${ }^{1}$, C. A. M. BALTAR ${ }^{1 *}$ e S. C. A. FRANÇA ${ }^{2}$ \\ ${ }^{1}$ Universidade Federal de Pernambuco (UFPE) \\ ${ }^{2}$ Centro de Tecnologia Mineral (CETEM) \\ camb@ufpe.br*
}

Artigo submetido em julho/2016 e aceito em julho/2016

DOI: $10.15628 /$ holos.2016.4823

\section{RESUMO}

A velocidade de sedimentação de partículas finas pode ser aumentada por meio de agregação por coagulação, aglomeração ou floculação. A floculação direta de partículas coloidais não é suficiente para aumentar significativamente a velocidade de sedimentação, pois o floco ainda pode ter pequeno tamanho. Portanto, a desestabilização de sistemas coloidais passa pela necessidade de uma pré-agregação. Nesse trabalho comparou-se a influência de uma pré-agregação, com um surfatante - dodecilamina (DDA) e com um sal - cloreto de alumínio (hexa)hidratado (CAH), na floculação de partículas coloidais de dióxido de titânio com uma poliacrilamida hidrolisada (PAMH). Os resultados foram avaliados em termos de turbidez do sobrenadante e compactação final do sedimento. A pré-agregação, com um eletrólito ou um surfatante, melhora significativamente a floculação com PAMH. A DDA proporciona a formação de pré-agregados maiores, menor turbidez do sobrenadante e sedimentos mais compactos, quando comparado com o $\mathrm{AlCl}_{3} \cdot 6 \mathrm{H}_{2} \mathrm{O}$.

PALAVRAS-CHAVE: pré-agregação, compactação do sedimento, floculação, coagulação, poliacrilamida.

\section{DESTABILIZATION OF A COLLOIDAL SUSPENSION OF TITANIUM DIOXIDE}

\begin{abstract}
The sedimentation rate can be increased by the processes of aggregation (coagulation, agglomeration, or flocculation). The direct flocculation of colloidal particles is not sufficient for the destabilization of a colloidal suspension. Due to the very small size of the colloids, the flocs can remain with very slow sedimentation rate. Therefore, a previous step of aggregation is necessary for an efficient flocculation. In this study it was compared the influence of pre-aggregation, with a surfactant dodecylamine (DDA) and a multivalent salt -
\end{abstract}

KEYWORDS: pre-aggregation, compaction of sediment, flocculation, coagulation, polyacrylamide. 


\section{INTRODUÇÃO}

O retorno de partículas coloidais na água de reuso das usinas de beneficiamento de minérios acarreta uma série de problemas na flotação, como: o aumento do consumo de reagentes (CHANDER, 1978; BALTAR, 1980), a contaminação do concentrado pelo arraste de partículas hidrofílicas (AKTAS et al., 2008; WANG et al., 2015), o recobrimento de partículas grossas ("slime coating") pelos finos (FENG et al., 2012), dentre outros fatores. Todos esses problemas podem ser reduzidos com uma maior eficiência do processo de agregação dos finos na etapa de espessamento.

A agregação é um processo físico-químico que desestabiliza a suspensão, facilitando a separação sólido-líquido. Na coagulação as forças superficiais de natureza atrativa superam as de natureza repulsiva, originando um "coágulo". Na floculação a agregação ocorre por meio do uso de polímeros de alto peso molecular, denominados floculantes, cujas moléculas adsorvem-se de forma simultânea na superfície de várias partículas originando um "floco" (BALTAR, 2010). Na indústria mineral a floculação é feita com poliacrilamidas neutras ou aniônicas (hidrolisada) com massa molar entre $10^{6}$ e $10^{7} \mathrm{~g} \cdot \mathrm{mol}^{-1}$ (MOODY, 1992). A floculação de partículas coloidais, no entanto, muitas vezes não resulta em uma sedimentação eficiente já que, devido ao pequeno tamanho das partículas, o floco continua com baixa velocidade de sedimentação. Nesses casos, há a necessidade de agregação prévia (BALTAR, 1997).

Este trabalho teve por objetivo estudar a influência da pré-agregação dos finos e, além disso, da natureza do pré-agregado formado, na eficiência da floculação de partículas coloidais de dióxido de titânio $\left(\mathrm{TiO}_{2}\right)$ com uma poliacrilamida aniônica parcialmente hidrolisada (PAMH).

\section{MATERIAIS E MÉTODO}

Utilizou-se uma amostra de dióxido de titânio coloidal $\left(\mathrm{TiO}_{2}\right)$, comercialmente conhecido como Hombitan Anatase (Figura 1), fabricado pela Sachtleben Chemie $\mathrm{GmbH}^{\circledR}$. Usou-se uma dodecilamina (DDA), fornecida pela SIGMA-ALDRICH; um cloreto de alumínio hexa-hidratado (CAH), da REAGEN; uma poliacrilamida parcialmente hidrolisada (PAMH), produzida pela KEMIRACYTEC. Como reguladores de $\mathrm{pH}$, foram usadas soluções de hidróxido de sódio $(\mathrm{NaOH})$ ou ácido clorídrico $(\mathrm{HCl})$, produzidos pela MERCK.

O condicionamento dos reagentes foi feito em um agitador EUROSTAR, digital, modelo Ika Werke; usou-se um granulômetro a laser modelo Mastersizer 2000 da MALVERN; um sistema ESA9800 da MATEC, para a determinação de potencial zeta e um turbidímetro, modelo B250, da MICRONAL.

Os testes de sedimentação foram realizados em provetas de $250 \mathrm{~mL}$, adaptadas com duas saídas laterais para a retirada de amostra a 3/5 e 4/5 da altura (da base ao topo). Foram avaliadas, em todos os testes, a variação de turbidez do sobrenadante em amostras coletadas durante a sedimentação, no intervalo de 30 a 180 segundos, e a altura de compactação do sedimento formado após $72 \mathrm{~h}$ de repouso. 
Os testes de floculação foram realizados para a polpa de $\mathrm{TiO}_{2}$, já com as partículas préagregadas com o eletrólito cloreto de alumínio hexa-hidratado (CAH) ou com o surfatante dodecilamina (DDA).

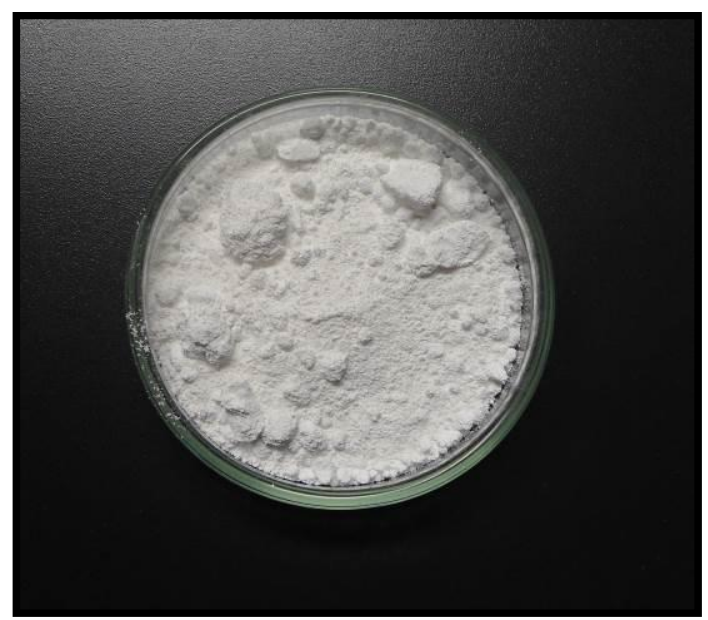

Figura 1 - Amostra de dióxido de titânio, Hombitan Anatase, produzido pela Sachtleben Chemie GmbH.

\section{RESULTADOS E DISCUSSÕES}

\subsection{Características da amostra}

As partículas de dióxido de titânio $\left(\mathrm{TiO}_{2}\right)$ apresentam granulometria média $\left(d_{50}\right)$ de 0,613 $\mu \mathrm{m}$ e área superficial de $1,18 \times 10^{4} \mathrm{~m}^{2} / \mathrm{kg}$, caracterizando-se, portanto, como uma amostra de tamanho coloidal (HUGHES, 1981). Segundo o fabricante, a amostra apresenta pureza acima de 99,0\% e, por isso, não houve necessidade da realização de uma etapa de purificação. O dióxido de titânio forma uma suspensão estável em água destilada. Após 30 dias de repouso a suspensão com concentração de sólidos de $24 \mathrm{~g} / \mathrm{L}$ manteve-se estável (sem sedimentação) em pH 7 (Figura 2).

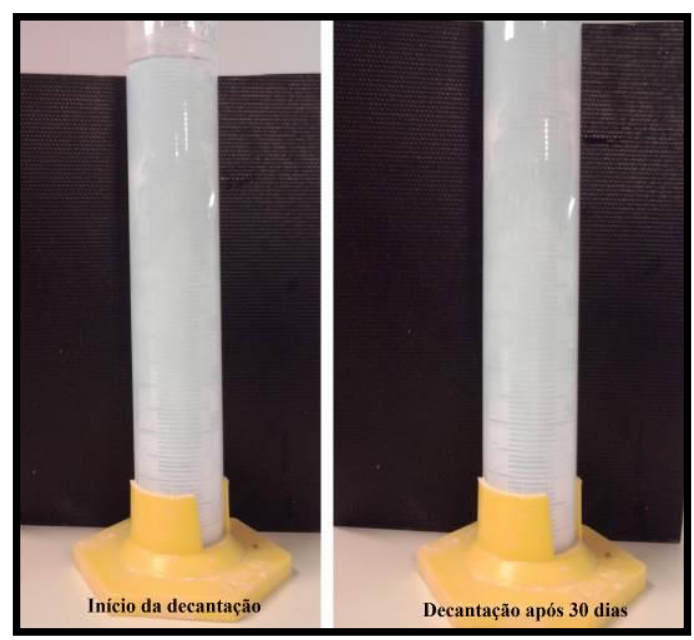

Figura 2 - Suspensão da amostra de anatásio, em água destilada, após 30 dias de repouso. 


\subsection{Floculação}

$\mathrm{O}$ aumento da concentração de $\mathrm{PAMH}$ na suspensão de $\mathrm{TiO}_{2}$ não é suficiente para proporcionar uma sedimentação perceptível ou uma redução significativa na turbidez do sobrenadante. Uma floculação eficiente só foi possível com o tratamento prévio da suspensão com o eletrólito $\left(\mathrm{AlCl}_{3} \cdot 6 \mathrm{H}_{2} \mathrm{O}\right)$ ou com surfatante (DDA). Os resultados apresentados na Figura 3 mostram que, sem a pré-agregação, a turbidez mínima ficou em torno de 100.000 NTU e que uma significativa redução da turbidez foi obtida com ambos os desestabilizantes. A pré-agregação com a DDA $\left(10^{-3} \mathrm{M}\right)$, sem a adição do floculante, reduziu a turbidez para valores em torno de $2.500 \mathrm{NTU}$. Uma posterior redução para valores inferiores a 500 NTU foi conseguida com a adição do floculante. $O$ aumento da concentração do DDA $\left(5 \times 10^{-3} \mathrm{M}\right)$ elevou o nível de turbidez da polpa. Isso, provavelmente, aconteceu devido ao início de um processo de pré-hemimicelização, que resultou em uma redução do tamanho do agregado. Na adsorção do floculante no pré-agregado formado por ação do $\mathrm{CAH}$, prevalecem as ligações de hidrogênio. Já na adsorção aos agregados hidrofóbicos formados pela DDA, há uma forte participação das interações hidrofóbicas entre as cadeias hidrocarbônicas do polímero e do surfatante.

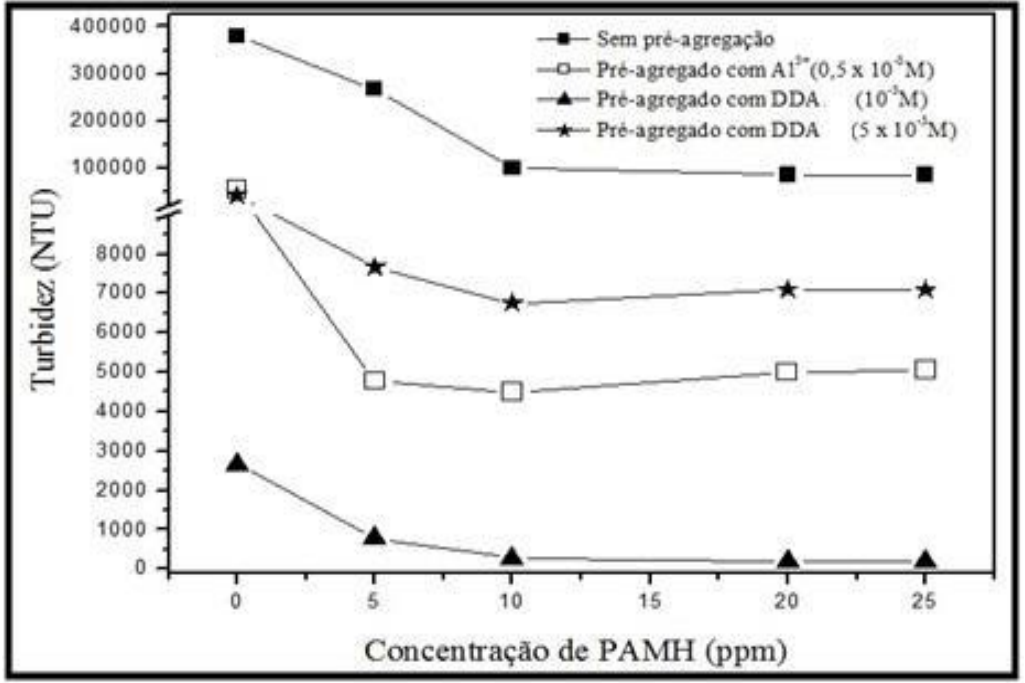

Figura 3 - Influência da concentração da PAMH na turbidez da suspensão de $\mathrm{TiO}_{2}$, após 30 segundos de repouso, em função do tratamento prévio da amostra.

A floculação após a pré-agregação com a DDA produz um sedimento mais compactado, quando comparado com o sedimento obtido após a pré-agregação com o $\mathrm{CAH}$, em todas as concentrações de PAMH testadas (Figura 4). Isso também foi observado por Baltar e Oliveira (1998) com uma suspensão de sílica coloidal e, provavelmente, se deve à presença de moléculas hidrofóbicas, no interior dos flocos, que promovem a expulsão da água retida, o que favorece a compactação do sedimento. A maior compactação é observada quando a pré-agregação é feita com DDA na concentração de $10^{-3} \mathrm{M}$, que corresponde a uma superfície próxima do ponto isoelétrico. A Tabela 1 apresenta o tamanho médio $\left(d_{50}\right)$ dos agregados formados na presença de PAMH em diferentes situações. Pode-se observar que, sem a pré-agregação, o tamanho do floco formado $(0,913 \mu \mathrm{m})$ é apenas $50 \%$ maior do que o tamanho médio das partículas individuais. Quando a floculação é precedida de agregação com DDA $\left(10^{-3} \mathrm{M}\right)$, no entanto, o tamanho médio do floco chega a 414,7 $\mu \mathrm{m}$ (691 vezes maior do que o tamanho médio das partículas coloidais). Os resultados não deixam dúvidas com relação à necessidade de uma agregação prévia à floculação. 


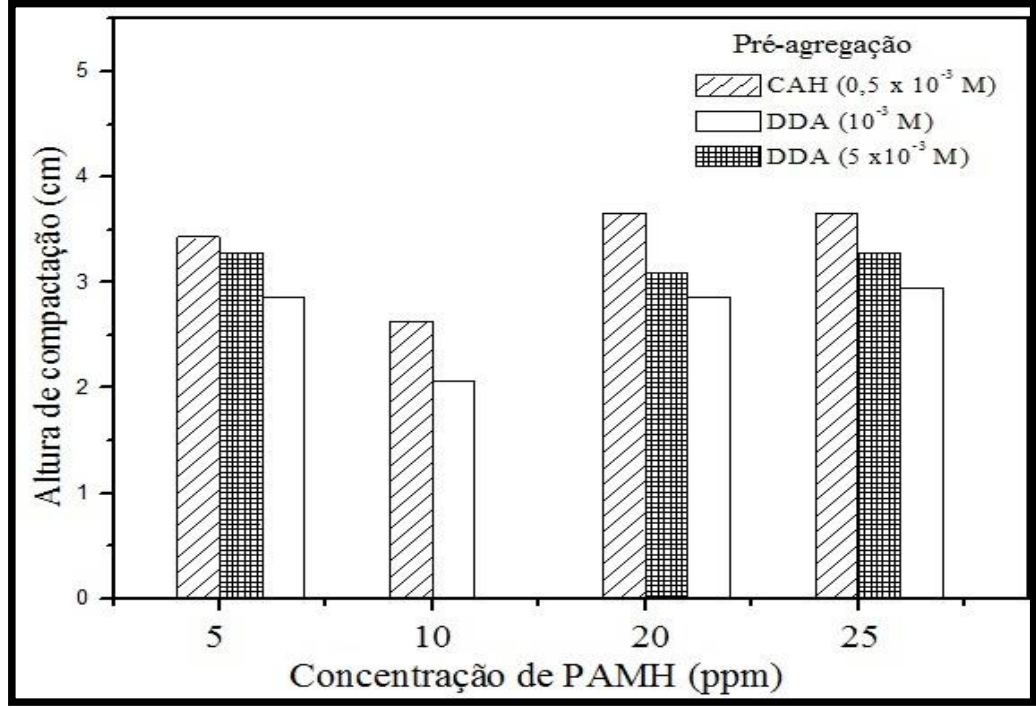

Figure 4 - Influência da concentração do floculante na altura final do sedimento após 72 horas.

Tabela 1: Diâmetro médio $\left(d_{50}\right)$ dos flocos obtidos em função do tratamento prévio.

\begin{tabular}{c|c|c|c}
\hline $\begin{array}{c}\text { Concentração de } \\
\text { PAMH }(\mathrm{ppm})\end{array}$ & $\begin{array}{c}\text { Concentração de } \mathrm{AlCl}_{3} \cdot 6 \mathrm{H}_{2} \mathrm{O} \\
\left(\times 10^{-3} \mathrm{M}\right)\end{array}$ & $\begin{array}{c}\text { Concentração de DDA } \\
\left(\times 10^{-3} \mathrm{M}\right)\end{array}$ & $\mathrm{d}_{50}(\mu \mathrm{m})$ \\
\hline 0 & - & - & 0,6 \\
10 & - & - & 0,9 \\
10 & - & 1,0 & 414,7 \\
10 & - & 5,0 & 19,1 \\
10 & 0,5 & - & 97,9 \\
\hline
\end{tabular}

\section{CONCLUSÃO}

A floculação apenas com a PAMH resulta em agregados que não têm tamanho suficiente para uma sedimentação eficiente. Por isso, a turbidez permanece alta. A desestabilização do sistema exige uma agregação prévia das partículas coloidais. A melhor redução de turbidez é conseguida após uma pré-agregação com DDA $\left(10^{-3} \mathrm{M}\right)$, e posterior floculação com PAMH (10 ppm). A partir dessa concentração de DDA a turbidez volta a subir. Isso é atribuído à formação de hemimicelas, ou seja, a reorientação das moléculas do surfatante na superfície das partículas, impedindo o crescimento do agregado. A adição de íons $\mathrm{Al}^{3+}$ provoca a compressão da dupla camada elétrica e o consequente abaixamento do potencial zeta das partículas para valores próximos ao ponto isoelétrico (pie), de modo a favorecer a formação de pré-agregados. Em ambos os casos, formam-se flocos grandes que sedimentam com facilidade. No entanto, a interação PAMH-DDA resulta em menor turbidez e maior compactação do sedimento. A maior compactação obtida com a DDA é atribuída à expulsão da água retida no floco. Considera-se que os resultados obtidos com a suspensão de dióxido de titânio possam ser estendidos para outros sistemas. 


\section{REFERÊNCIAS}

1. AKTAS, Z.; CILLIERS, J.J.; BANFORD, A.W., 2008. Dynamic froth stability: Particle size, airflow rate and conditioning time effects. International Journal of Mineral Processing, 87, 65-71.

2. BALTAR, C.A.M., 1980. Aproveitamento de Minério Oxidado de Zinco com Baixo Teor por Flotação. Dissertação de Mestrado, Programa de Engenharia Metalúrgica e de Matérias da COPPE, Universidade Federal do Rio de Janeiro. Rio de Janeiro, 226 p.

3. BALTAR, C. A. M.,1997. Influência da interação polímero-surfatante na floculação de uma sílica coloidal com poliacrilamida. Tese (Doutorado) - Universidade do Rio de Janeiro, Rio de Janeiro, $198 \mathrm{p}$.

4. BALTAR, C.A.M., 2010. Processos de Agregação. In.: Tratamento de Minérios - 5a Edição. Luz, A.B.; Sampaio, J.A.; França, S.C.A. (editores). CETEM/MCT. Capítulo 13, p. 557-594.

5. CHANDER, S., 1978. Recent developments in floatability of fine particles - A review. Transactions of The Indian Institute de Metals, 31 (1), 12-19.

6. FENG, B.; LU, Y.; FENG, Q.; LI, H., 2012. Solution chemistry of sodium silicate and implications for pyrite flotation. Industrial \& Engineering Chemistry Research, 51, 12089-12094.

7. HUGHES, M.A., 1981. Coagulation and Flocculation. In.: Solid-Liquid Separation - 2a ed. Svarovsky, L. (editor). Butterworths.

8. MOODY, G., 1992. The use of polyacrylamides in mineral processing. Minerals Engineering, 5 (3-5), 479-492.

9. WANG, L.; PENG, Y.; RUNGE, K.; BRADSHAW, D., 2015. A review of entrainment: mechanisms, contributing factors and modelling in flotation. Minerals Engineering, 70, 77-91. 